\title{
Time-Resolved Fluorescence Study of the Dissociation of FMN from the Yellow Fluorescence Protein from Vibrio fischeri
}

\author{
Antonie J. W. G. Visser ${ }^{1}$, Arie van Hoek ${ }^{1}$, Nina V. Visser ${ }^{1}$, Yongho Lee ${ }^{2}$ and Sandro Ghisla*2 \\ 'Department of Biochemistry, Agricultural University, Wageningen, the Netherlands and \\ ${ }^{2}$ Faculty of Biology, University of Konstanz, Konstanz, Germany
}

\begin{abstract}
Time-resolved fluorescence spectroscopy of the flavin mononucleotide (FMN) prosthetic group of the yellow fluorescence protein (YFP) from Vibrio fischeri has provided quantitative, thermodynamic information on the FMN-apoYFP equilibrium in aqueous buffer. In diluted aqueous solution two fluorescent species could be identified by distinct fluorescence lifetimes and rotational correlation times originating from free- and protein-bound FMN. Quantitation of the amounts of free and bound FMN in progressively larger dilutions of YFP in aqueous buffer yielded a dissociation constant of $0.40 \mu M$ for the FMN-apoprotein complex at $20^{\circ} \mathrm{C}$. The single fluorescence lifetime of YFP-bound FMN is very long (7.6 $\mathrm{ns}$ at $20^{\circ} \mathrm{C}$ ), suggesting a binding environment in which maximal emission is provided commensurate with its function as a bioluminescent emitter. The single correlation time of $14.8 \mathrm{~ns}\left(20^{\circ} \mathrm{C}\right)$ is in agreement with a rigid binding site that rotates together with the whole, hydrated protein. Using a different technique we have obtained the same results as reported by others (G. Sirokman, T. Wilson and J. W. Hastings, Biochemistry 34, 13074-13081, 1995; V. N. Petushkov, B. G. Gibson and J. Lee, Biochem. Biophys. Res. Commun. 211, 774-779, 1995).
\end{abstract}

\section{INTRODUCTION}

The yellow emission of a strain of Vibrio fischeri bioluminescent bacteria has been identified with a yellow fluorescence protein (YFP) $\dagger(1,2)$. This strongly emitting protein turned out to be a flavoprotein with flavin mononucleotide (FMN) $(3,4)$. In addition, a closely related protein containing riboflavin as the chromophore has been identified $(5,6)$.

The similarity of YFP with the function and properties of the lumazine protein isolated from blue light-emitting bioluminescent photobacteria is evident (reviewed in Lee et al. (7)). The prosthetic group of this protein, 6,7-dimethyl-8ribityllumazine, has excellent fluorescent properties (high

\footnotetext{
*To whom correspondence should be addressed at: Universität Konstanz, Fakultät für Biologie, Universitätsstrasse 10, Postfach 5560 M644, D-78464 Konstanz, Germany. Fax: 7531-882291.

$\dagger$ Abbreviations: FMN, flavin mononucleotide; YFP, yellow fluorescent protein.
}

fluorescence quantum yield, long fluorescence lifetime) enabling the investigation of ligand-protein and protein-protein interaction and protein dynamics using thermodynamically ideal, diluted protein solutions $(8,9)$. The YFP has these convenient properties as well $(10,11)$. It is one of the flavoproteins with the highest fluorescence quantum yield occurring in nature and it is characterized by a peculiarly red-shifted fluorescence spectrum as compared to that of FMN in aqueous solution.

In this study we have employed sensitive time-resolved polarized fluorescence spectroscopy with the aim of characterizing the equilibrium binding between FMN and the apoprotein of YFP and to investigate the dynamic properties of the flavin binding site. Our binding results are fully in line with the data and conclusions of Sirokman et al. (10), which were derived from steady-state fluorescence anisotropy measurements.

\section{MATERIALS AND METHODS}

The YFP was isolated from $V$. fischeri strain $\mathrm{Y} 1$ according to a procedure described previously (3). The major fraction corresponding to the FMN bound protein (6) was collected and the identity of the cofactor FMN was verified by HPLC. Prior to the time-resolved fluorescence measurements, a small amount of the protein stock solution was put on a desalting column (Econo-Pac ${ }^{\circledR}$ 10DG, Bio-Rad, Hercules, CA) and eluted with the desired buffer (for dilution experiments at $20^{\circ} \mathrm{C}$ : $0.1 \mathrm{M}$ Tris $\mathrm{HCl}, \mathrm{pH} 7.5$; for experiments at different temperatures: $0.05 \mathrm{M}$ potassium phosphate, $\mathrm{pH} 7.5$ ).

Fluorescence decay and fluorescence anisotropy decay were measured using a laser set-up and time-correlated single photon counting essentially as described elsewhere $(12,13)$. The excitation wavelength was $465 \mathrm{~nm}$ and the emission was selected by a KV500 cutoff (Schott, Mainz, Germany) and $557.6 \mathrm{~nm}$ interference filter (Schott, $10 \mathrm{~nm}$ bandpass). For linking several decay experiments to recover common decay parameters, the second-generation global analysis software program (obtained from Globals Unlimited ${ }^{\text {(m) }}, \mathrm{Ur}$ bana, IL, USA) was used (14). A detailed error analysis of the parameters at the $67 \%$ confidence level was performed as described in Beechem et al. (14). The following equations were used for the evaluation of average fluorescence lifetimes, molecular size and binding equilibria.

The second-order average fluorescence lifetime, $\langle\tau\rangle$, is given by

$$
\langle\tau\rangle=\frac{\sum_{i=1}^{2} \alpha_{i} \tau_{i}^{2}}{\sum_{i=1}^{2} \alpha_{i} \tau_{i}} .
$$

The long rotational correlation time is proportional to the size of the protein. For spherical proteins with the fluorescent group rigidly bound, a simple, empirical formula relates the rotational correlation 
time to the molecular mass $M_{r}$, the partial specific volume $\bar{V}$, the viscosity $\eta$, the temperature $T$ and the degree of hydration $h$ :

$$
\phi=\frac{M_{r} \eta(\bar{v}+h)}{R T} .
$$

The equilibrium constant for the dissociation of FMN from YFP can be obtained from fluorescence parameters. According to Sirokman et al. (10) and Spencer and Weber (15), the following equations are used for determination of $K_{\mathrm{D}}$ from steady-state anisotropies $<\mathrm{r}>$ :

$$
f_{\mathrm{B}}=\frac{\langle\mathrm{r}\rangle-\langle\mathrm{r}\rangle_{\mathrm{F}}}{\left(\langle\mathrm{r}\rangle_{\mathrm{B}}-\langle\mathrm{r}\rangle\right) \mathrm{R}+\left(\langle\mathrm{r}\rangle-\langle\mathrm{r}\rangle_{\mathrm{F}}\right)}
$$

where $f_{B}$ is the fraction of bound $F M N$, the subscripts $B$ and $F$ denote bound and free FMN and $R$ is the ratio of the fluorescence quantum yields of bound and free FMN. ( $R$ can be set equal to the ratio of fluorescence lifetimes or better, as reasoned below, to a factor of 1.22.) The dissociation constant is then obtained as

$$
K_{\mathrm{D}}=\frac{[\mathrm{YFP}]_{\mathrm{total}}\left(1-f_{\mathrm{B}}\right)^{2}}{f_{\mathrm{B}}} .
$$

The steady-state anisotropies for free and bound FMN were obtained from the Perrin equation:

$$
\frac{\mathrm{r}_{0}}{\langle\mathrm{r}\rangle}=1+\frac{\tau}{\phi}
$$

where $r_{0}$ is the fundamental anisotropy, $\tau$ the fluorescence lifetime and $\phi$ the rotational correlation time.

\section{RESULTS AND DISCUSSION}

\section{Dilution experiments}

We started with a concentration of $5.76 \mu M$ YFP and made two-fold dilutions in aqueous buffer so as to reach five additional YFP concentrations. The six total fluorescence decays were analyzed simultaneously using a triple exponential function in which the two longer lifetime components were linked over all decay curves. The shortest lifetime was required to obtain an optimum fit and was left as an unconstrained parameter in the fit. Its value was about $0.1-0.2 \mathrm{~ns}$, and its contribution was minor. The nature of this short life-

ne component is still unclear. A selection of the results (hree decay curves) is shown in Fig. 1. The weighted residuals are presented as well to illustrate that an optimal fit was obtained. When YFP is diluted the equilibrium of FMN binding to the apoprotein is shifted to dissociation of FMN. This is manifested by a faster decay because free FMN has a shorter lifetime of $4.7 \mathrm{~ns}(16,17)$ as compared to the 7.6 ns lifetime of bound FMN (see curve 3 in Fig. 1). At the highest concentration of $5.76 \mu M$ there is only a tiny amount of dissociated FMN present as indicated by the predominantly longer decay of bound FMN (see curve 1 in Fig. 1). The 7.6 ns lifetime component is the longest flavin fluorescence lifetime ever observed in flavoproteins and its presence reflects an FMN binding site where no quenching groups like cysteine or aromatic amino acids are present in the vicinity of the flavin ring. This binding site is optimized for fluorescence, in full agreement with the function of YFP in emitting bioluminescence with high efficiency. Petushkov et al. (6) also reported a lifetime of $7.6 \mathrm{~ns}$ but at $0^{\circ} \mathrm{C}$.

When the fluorescence decays were analyzed separately, it was noted that the long lifetime was correctly recovered but the shorter lifetime turned out to be in the range 3.6-3.8 ns, which is shorter than the published FMN fluorescence lifetime of $4.7 \mathrm{~ns}$ at room temperature $(16,17)$. This certainly has to do with the difficulty in the unambiguous determi-
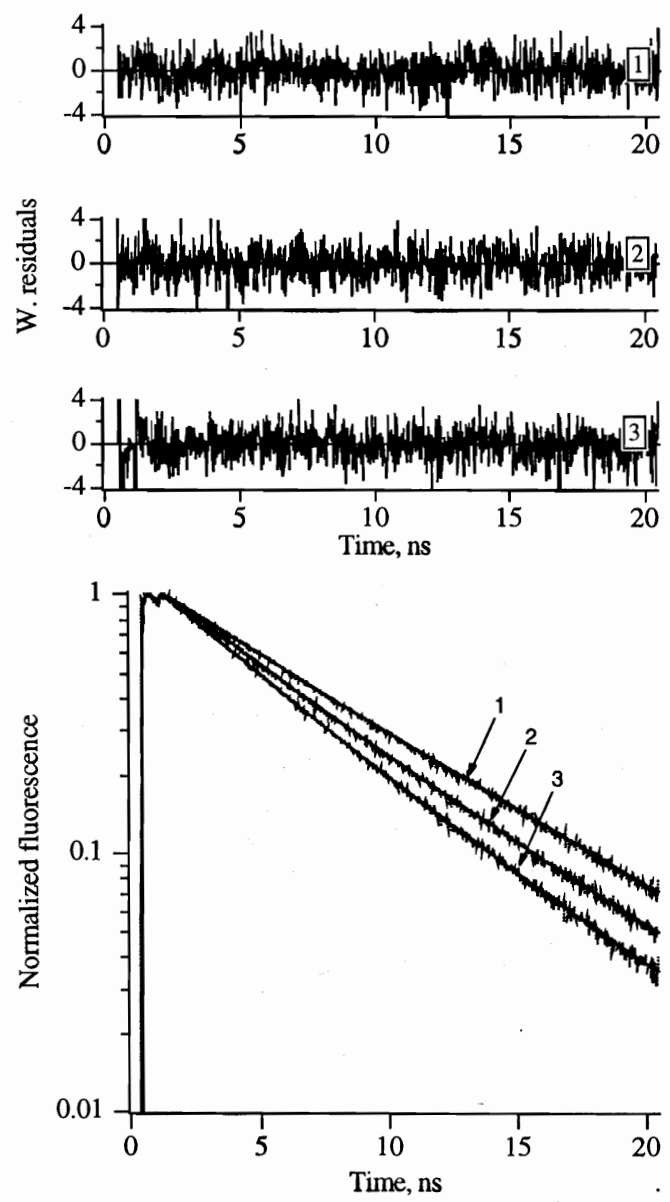

Figure 1. Experimental and fitted total fluorescence decay curves of YFP in aqueous Tris/ $\mathrm{HCl}$ buffer, $\mathrm{pH} 7.5$, at $20^{\circ} \mathrm{C}$. Three sets of curves are presented corresponding with YFP concentrations of 5.76 $\mu M$ (curve 1), $0.72 \mu M$ (curve 2) and $0.18 \mu M$ (curve 3 ). The three sets of experimental data were globally analyzed together with three other data sets and optimized parameter values $\left(\alpha_{i}, \tau_{i}, i=1,2\right)$ are collected in Table 1 . The two lifetimes were considered common parameters and therefore linked over all data sets (one is assigned to free FMN and the other to bound FMN). The quality of the fits is indicated by the weighted residuals between experimental and fitted curves. To obtain such an excellent fit it was necessary to include a third subnanosecond component of low amplitude, the parameter values of which were allowed to adjust without zonstraints. The channel width was 20 ps and 1024 channels were used.

nation of relatively close fluorescence lifetimes. Global analysis turned out to be an appropriate method to determine the fluorescence lifetimes of free and bound FMN separately. In Table 1 the fractional amplitudes arising from the $7.6 \mathrm{~ns}$ component $\left(\alpha_{2}\right)$ relative to those, of the $4.4 \mathrm{~ns}$ component $\left(\alpha_{1}\right)$ as obtained from global analysis are collected (the contribution of the 0.1-0.2 ns component was ignored). In order to make comparison with the time correlated single photon counting lifetimes as determined previously (10), the second-order average fluorescence lifetimes, $\langle\tau\rangle$, obtained from Eq. 1, have been listed as well in Table 1. The average fluorescence lifetimes are in good agreement with the ones obtained by Sirokman et al. (10).

The separate parallel and perpendicular polarized intensity decay curves of the six YFP samples can be globally analyzed as well to yield rotational correlation times of free and 
Table 1. Fluorescence decay and anisotropy decay parameters of YFP at different concentrations as obtained from global analysis*

\begin{tabular}{llccccccccc}
\hline $\begin{array}{l}\text { Concen- } \\
\text { tration }(\mu M)\end{array}$ & $\alpha_{1}$ & $\begin{array}{c}\tau_{1} \dagger \\
(\mathrm{ns})\end{array}$ & $\alpha_{2}$ & $\begin{array}{c}\tau_{2} \ddagger \\
(\mathrm{ns})\end{array}$ & $\begin{array}{c}<\tau>\S \\
(\mathrm{ns})\end{array}$ & $\beta_{1}$ & $\begin{array}{c}\phi_{1} \| \\
(\mathrm{ns})\end{array}$ & $\beta_{2}$ & $\begin{array}{c}\phi_{2} \# \\
(\mathrm{~ns})\end{array}$ \\
\hline 5.76 & 0.08 & 4.4 & 0.92 & 7.6 & 7.4 & 0.02 & 0.15 & 0.29 & 14.8 & 0.157 \\
2.88 & 0.31 & 4.4 & 0.69 & 7.6 & 6.9 & 0.09 & 0.15 & 0.25 & 14.8 & 0.138 \\
1.44 & 0.29 & 4.4 & 0.71 & 7.6 & 7.0 & 0.10 & 0.15 & 0.25 & 14.8 & 0.140 \\
0.72 & 0.34 & 4.4 & 0.66 & 7.6 & 6.8 & 0.17 & 0.15 & 0.19 & 14.8 & 0.108 \\
0.36 & 0.64 & 4.4 & 0.36 & 7.6 & 6.0 & 0.22 & 0.15 & 0.13 & 14.8 & 0.081 \\
0.18 & 0.69 & 4.4 & 0.31 & 7.6 & 5.8 & 0.26 & 0.14 & 0.11 & 14.8 & 0.071 \\
\hline
\end{tabular}

*All fluorescence lifetimes were linked over six experiments; all rotational correlation times were linked over 12 experiments (parallel and perpendicular decay curves for each sample). The preexponential factors $\alpha_{i}$ and $b_{i}(i=1,2)$ were the unconstrained parameters. The temperature was $293 \mathrm{~K}$.

†Lower and upper bounds as determined from a rigorous error analysis at a $67 \%$ confidence level are for this parameter: $4.1<4.4<4.7 \mathrm{~ns}$.

$\ddagger$ Lower and upper bounds for this parameter: $7.2<7.6<8.3 \mathrm{~ns}$.

$\S$ Second-order average fluorescence lifetime.

Lower and upper bounds for this parameter: $0.08<0.15<0.23 \mathrm{~ns}$.

\#Lower and upper bounds for this parameter: $13.5<14.8<16.0 \mathrm{~ns}$.

IISteady-state anisotropy.

${ }^{* *}$ Quality of fit of individual experiment of global fluorescence decay analysis. For the global fit $\chi^{2}=1: 13$ and for the global fit in case of anisotropy decay analysis $\chi^{2}=1.22$.
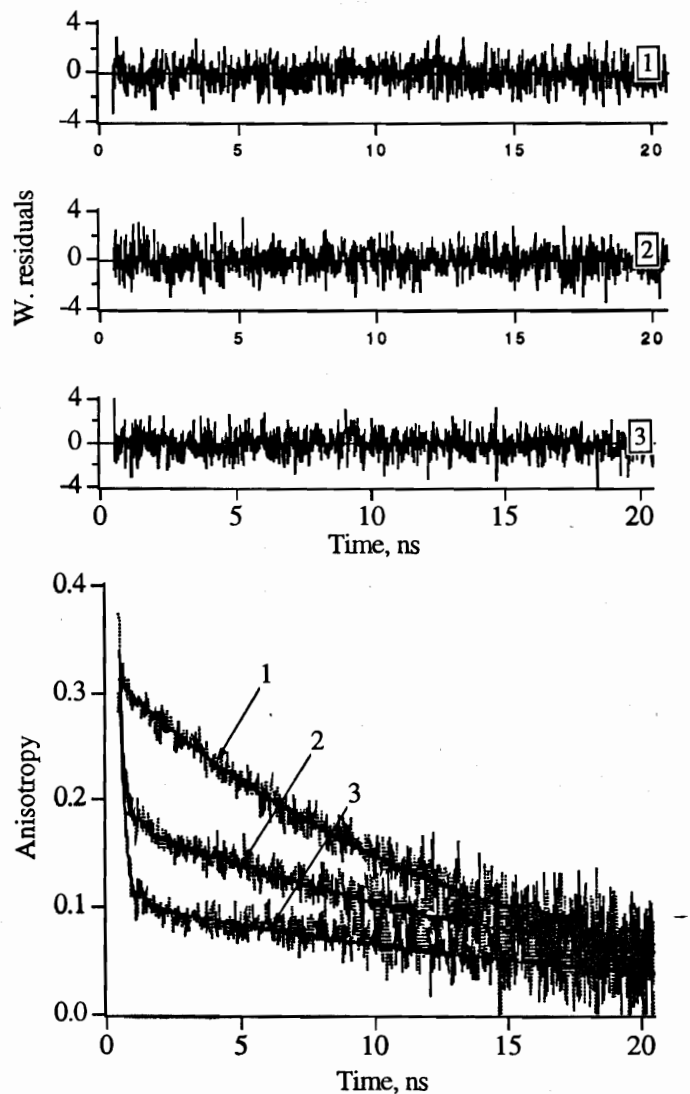

Figure 2. Experimental and fitted fluorescence anisotropy decay curves of the same YFP samples as detailed in the legend to Fig. 1. The anisotropy decay curves were constructed from the parallel and perpendicular polarized decay curves that were globally analyzed with other experimental data sets to yield the parameter values $\left(\beta_{\mathrm{i}}\right.$, $\left.\phi_{i}, i=1,2\right)$ collected in Table 1 . Two common correlation times were linked over all data sets: one short correlation time arises from unbound FMN and a much longer correlation time arises from FMN tightly bound in YFP. The weighted residuals are obtained as described in Spencer and Weber (15), and they illustrate the good quality of the fits. bound FMN and their fractional contributions (the fluorescence decay parameters were fixed in this analysis). Three examples are presented in Fig. 2, in which experimental and fitted anisotropies were constructed from the individual polarized decay curves and the weighted residuals calculated according to a published procedure (18). The fits were excellent. From a global analysis, common rotational correlation times of $0.15 \mathrm{~ns}$ and $14.8 \mathrm{~ns}$ were found. The long correlation time arises from YFP-bound FMN and is apparent from the slow anisotropy decay in the most concentrated sample (see curve 1 in Fig. 2). A long correlation time of similar magnitude has also been found recently by Sirokman et al. (10) and by Petushkov et al. (6) at $0^{\circ} \mathrm{C}$. The short correlation time has also been assigned previously to free FMN (19). Its presence is very clear as a very fast decay in the more dilute samples (see curve 3 in Fig. 2). The most concentrated sample of YFP (curve 1 in Fig. 2) has some interesting anisotropy properties. The fundamental anisotropy is distinctly lower $\left(r_{0}=0.31\right)$ than for other flavoproteins $\left(r_{0}=0.36-0.38\right)(20,21)$. This suggests that the angle $\delta$ between absorption and emission transition moments in the isoalloxazine plane is larger when FMN is bound in YFP $(\delta$ $=23^{\circ}$ as compared to $\delta=15^{\circ}$ of FAD in lipoamide dehydrogenase). Furthermore, there is no independent rotational mobility of bound FMN indicating that the tightly bound prosthetic group rotates together with the whole protein. This tight association of the prosthetic group has also been observed in the closely related lumazine protein (7). All relevant anisotropy parameters $\left(\beta_{\mathrm{i}}, \phi_{\mathrm{i}}, \mathrm{i}=1,2\right)$ retrieved from global analysis have been collected in Table 1. Again, in order to make comparisons with previously determined steady-state anisotropies (10), we have determined $\langle\mathrm{r}\rangle$ from the integrated experimental anisotropy decays and have listed these values in Table 1.

\section{Experiments at varying temperatures}

In the preceding paragraph it was demonstrated that the binding equilibrium can be monitored by determining the contributions of fluorescence lifetimes and rotational corre- 
Table 2. Fluorescence decay and anisotropy decay parameters of $1.0 \mathrm{mM}$ YFP at different temperatures

\begin{tabular}{|c|c|c|c|c|c|c|c|c|c|c|c|}
\hline 278 & 0.39 & 4.7 & 0.61 & $\begin{array}{c}8.1 \\
(8.0-8.2)\end{array}$ & 7.2 & 0.11 & $\begin{array}{c}0.37 \\
(0.20-0.70)\end{array}$ & 0.24 & $\begin{array}{c}28.3 \\
(26.0-32.0)\end{array}$ & 0.161 & 1.07 \\
\hline 285 & 0.38 & 4.6 & 0.62 & $\begin{array}{c}7.8 \\
(7.8-8.1)\end{array}$ & 6.9 & 0.12 & $\begin{array}{c}0.34 \\
(0.19-0.58)\end{array}$ & 0.23 & $\begin{array}{c}20.8 \\
(19.3-22.6)\end{array}$ & 0.135 & 1.06 \\
\hline 292 & 0.42 & 4.4 & 0.58 & $\begin{array}{c}7.6 \\
(7.5-7.8)\end{array}$ & 6.6 & 0.14 & $\begin{array}{c}0.21 \\
(0.12-0.34)\end{array}$ & 0.21 & $\begin{array}{c}15.7 \\
(14.7-17.0)\end{array}$ & 0.106 & 1.11 \\
\hline
\end{tabular}

*Lifetimes were fixed at given values during analysis.

†Values in parentheses are the lower and upper bounds as determined from a rigorous error analysis at a 67\% confidence level (see legend to Table 1).

$\ddagger$ Second-order average fluorescence lifetime.

$\S$ Steady-state anisotropy.

॥Quality of fit of global analysis of parallel and perpendicular fluorescence decay curves (see legend to Table 1).

lation times in the respective decays. The same approach can be used to find out whether the binding equilibrium shows a temperature dependence. A concentration of 1.0 $\mu M$ YFP was chosen because a measurable amount of dissociated FMN is present. Measurements were performed at three temperatures and data analysis was performed on the decay curves at a single temperature. The results are presented in Table $2\left(\alpha_{i}, \tau_{i}, \beta_{i}, \phi_{i}, i=1,2\right)$. The interesting observation is that the preexponential factors and the long rotational correlation time are somewhat dependent on temperature. The dependence of the amplitudes $\alpha_{i}$ and $\beta_{i}$ on temperature suggests a possible shift in the binding equilibrium, which will be addressed in the following section. The longer rotational correlation time is proportional to the size of YFP. Substituting the appropriate values $\left(\overline{\mathrm{v}}=0.74 \mathrm{~cm}^{3} / \mathrm{g}, \mathrm{h}=0.4 \mathrm{~cm}^{3} /\right.$ $\mathrm{g}, \eta=1.027 \mathrm{cp}, \mathrm{T}=292 \mathrm{~K}, \mathrm{M}=22.7 \mathrm{kDa}$ ) into Eq. 2 yields a correlation time of $11.0 \mathrm{~ns}$, which is somewhat shorter than the determined $15.7 \mathrm{~ns}\left(\phi_{2}\right.$ at $292 \mathrm{~K}$ in Table 2 ). The longer correlation time may arise from some ellip-
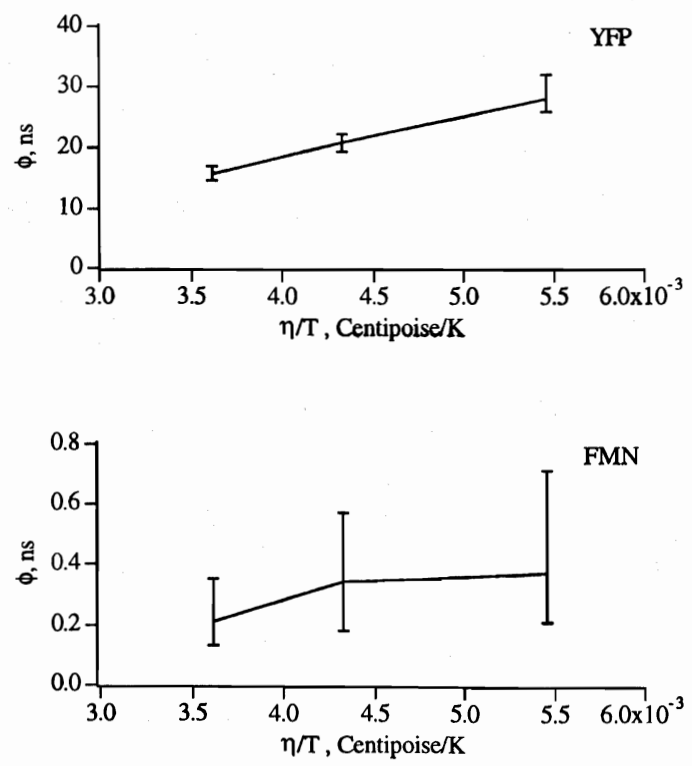

Figure 3. Rotational correlation times of free FMN and YFP plotted as a function of $\eta / T$. Values of correlation times and error bars are given in Table 2 . soidal shape of the protein as has been observed previously for lumazine protein (21). From Eq. 2 it can also be seen that the correlation time should be proportional to $\eta / T$. This is confirmed for YFP as illustrated in Fig. 3. It is unlikely that YFP dimers are formed at lower temperature (10) under our conditions because the plot of correlation time versus $\eta$ / $\mathrm{T}$ would deviate from linearity in the case of dimers. The errors in the rotational correlation time of unbound FMN are too large to draw similar conclusions as for bound FMN, but it should be realized that the time-scale of the experiment was designed for protein rotation and not for subnanosecond FMN rotation (see Fig. 2).

\section{Determination of equilibrium dissociation constants}

Given the relative contributions of decay times in both fluorescence decay and anisotropy decay, it is possible to determine the equilibrium dissociation constant $\left(K_{\mathrm{D}}\right)$ from these data. One should be aware, however, of the excitation wavelength $(465 \mathrm{~nm})$ and emission wavelength $(557.6 \mathrm{~nm})$ used in the decay experiments. The extinction coefficients at $465 \mathrm{~nm}$ for free FMN are $10400 \mathrm{~cm}^{-1} M^{-1}$ and $11800 \mathrm{~cm}^{-1}$ $M^{-1}$ for YFP (24). This implies that, in a mixture of bound and unbound FMN, there is a preference for exciting bound FMN by a factor of 1.13 . The same arguments apply for the emission wavelength: at $557.6 \mathrm{~nm}$ the fluorescence intensity of YFP is 1.08 times larger than that of FMN in water. The combination of the two factors implies that the relative concentration of bound FMN (given by $\alpha_{2}$ in Tables 1 and 2) must be divided by a factor of 1.22 to provide the real fractional concentration in the equilibrium mixture. In the case of anisotropy, the fundamental anisotropy is lower than for free FMN. Therefore, the preexponential factor that belongs to YFP (given by $\beta_{2}$ in Tables 1 and 2) has to be scaled to 0.31 to obtain the relative contribution of YFP in the anisotropy decay, and this value has to be scaled again by the factor of 1.22. In this way we have evaluated for each experiment (except for the $5.76 \mu M$ sample) the $K_{\mathrm{D}}$ value, and these data are collected in Table 3 . It is noted that the $K_{\mathrm{D}}$ values obtained from fluorescence decay and anisotropy decay are in fair agreement. Averaging the dissociation constants determined from fluorescence decay analysis of YFP at different concentrations (except the one at $2.88 \mu M$, which seems to be an outlier) leads to $K_{\mathrm{D}}=0.43 \pm 0.12 \mu M$. 
Table 3. Equilibrium dissociation constants describing the binding of FMN to the apoprotein of YFP*

\begin{tabular}{lcc}
\hline $\begin{array}{l}\text { Concentration } \dagger \\
(\mu M)\end{array}$ & $\begin{array}{c}K_{\mathrm{D}} \ddagger \\
(\mu M)\end{array}$ & $\begin{array}{c}\mathrm{K}_{D} \S \\
(\mu M)\end{array}$ \\
\hline 2.88 & 0.96 & 0.50 \\
1.44 & 0.43 & 0.25 \\
0.72 & 0.28 & 0.35 \\
0.36 & 0.61 & 0.45 \\
0.18 & 0.39 & 0.31 \\
Temperature & & $\Delta \mathrm{G}^{0}$ \\
$(\mathrm{~K})$ & $K_{\mathrm{R}}^{\#}$ & $\left(\mathrm{~kJ} \mathrm{~mol}^{-1}\right)$ \\
\hline 278 & $(\mu M)$ & 34.7 \\
285 & 0.30 & 34.4 \\
292 & 0.50 & 34.0 \\
\hline
\end{tabular}

* See text for details.

†Temperature $293 \mathrm{~K}$.

$\ddagger K_{\mathrm{D}}$ from fluorescence decay analysis.

$\S K_{\mathrm{D}}$ from anisotropy decay analysis.

Concentration $1.0 \mu M$.

$\# K_{\mathrm{D}}$ from steady-state anisotropy.

IIStandard free energy change for the dissociation reaction; from $\Delta \mathrm{G}^{0}$ $=-\mathrm{RT}$ in $\ln K_{\mathrm{D}}$.

Averaging all dissociation constants determined from anisotropy decay analysis results in $K_{\mathrm{D}}=0.37 \pm 0.10 \mu \mathrm{M}$. The average of nine experiments yields $K_{\mathrm{D}}=0.40 \pm 0.11$ $\mu M$. This value is higher than the dissociation constant found earlier for YFP using steady-state anisotropy $\left(K_{\mathrm{D}}=0.17 \mu M\right.$ at $\left.20^{\circ} \mathrm{C}(10)\right)$. It is also higher than the one of lumazine protein from Photobacterium phosphoreum, which binds the lumazine ligand with $K_{\mathrm{D}}=0.16 \mu M$ at $20^{\circ} \mathrm{C}$ (8).

In order to assess why these differences in $K_{\mathrm{D}}$ show up let us compare both methods and check whether they are internally consistent. For instance, at $1.44 \mu M$ YFP, $<\mathrm{r}\rangle=$ 0.140 (Table 1), $\mathrm{R}=1.22,<\mathrm{r}>_{\mathrm{F}}=0.012,<\mathrm{r}>_{\mathrm{B}}=0.205$, $\mathrm{f}_{\mathrm{B}}=0.61$ and $K_{\mathrm{D}}=0.36 \mu \mathrm{M}$. This value is almost exactly the average of the two equilibrium dissociation constants obtained from lifetimes and correlation times as given in Table 3. In other words, the results are internally consistent, because both decay times are used in the Perrin equation (Eq. 5). The choice of the value of $\mathrm{R}$ is determining for the exact value of $K_{\mathrm{D}}$. If we take $\mathrm{R}$ as the ratio of lifetimes ( $\mathrm{R}$ $=1.73$ ), then $\mathrm{f}_{\mathrm{B}}=0.53$ and $K_{\mathrm{D}}=0.17 \mu M$, the value of which is identical to that found by Sirokman et al. (10).

In Table 3 the equilibrium dissociation constants as retrieved from Eqs. 3-5 have been presented as a function of temperature. The lower the temperature the smaller the $K_{\mathrm{D}}$ value, which suggests a stronger complex between FMN and apo-YFP. In Table 3 the standard free energy for the dissociation reaction is given at each temperature. A van't Hoff plot of $\ln K_{\mathrm{D}}$ versus $1 / \mathrm{T}$ gives the standard free enthalpy $\Delta \mathrm{H}^{0}$ (48 kJ/mol) and entropy $\Delta S^{0}$ (48 eu) of the dissociation reaction. These values are somewhat lower than evaluated earlier $(8,10)$ but are on the same order of magnitude.

Weber (22) has discussed the forces involved in binding ligands (like FMN) to proteins. From the known three-dimensional structures, it is observed that the ligand is usually bound to the protein through interactions involving almost all atoms of the ligand. The forces are mainly originating from van der Waals contacts, electrostatic interactions (both permanent charges and hydrogen bonding) and differences in solvation between free and bound interaction partners. It was illustrated that a tiny displacement of charge $(0.1 \AA)$, as in a salt bridge, can yield an energy change of at least 5.5 $\mathrm{kJ} \mathrm{mol}^{-1}$ (22), which is even much larger than observed in YFP. Compensating contributions to the interaction forces as a function of temperature may play a role in the case of YFP. On the other hand the stronger complex at lower temperatures suggests that hydrophobic interactions provide the driving force for the stability of the complex (16).

In conclusion our results point to the unique fluorescence properties of YFP, which are undoubtedly related to its role in bacterial luminescence. Thus, interactions with the protein will minimize the processes leading to radiationless decay and maximize the probabilities of productive interaction between YFP itself and luciferase, in particular between the chromophores bound to these proteins. Protein-protein interaction can then result in a shielding from collisions with solvent and in a specific mode of docking, thereby preventing vibrations leading to internal conversion and loss of fluorescence quantum yield. Such a behavior shows a clear similarity with that of another photoprotein involved in bacterial bioluminescence, namely lumazine protein (7). The interaction between lumazine protein and luciferase does not lead to any quenching of the lumazine excited state. Therefore, the binding sites in both photoproteins are optimized for their function in emitting light with maximum quantum efficiency. The decay times (fluorescence lifetime and rotational correlation times) are in agreement with those measured by Petushkov et al. (6) and estimated by Sirokman et al. (10) from steady-state fluorescence anisotropy measurements and single photon counting fluorometry. Our conclusions concerning the binding equilibrium essentially confirm those of Sirokman et al. (10).

\section{REFERENCES}

1. Ruby, E. G. and K. H. Nealson (1977) A luminous bacterium that emits yellow light. Science 196, 432-434.

2. Leisman, G. and K. H. Nealson (1982) Characterization of a yellow fluorescent protein from Vibrio (Photobacterium) fischeri. In Flavins and Flavoproteins (Edited by V. Massey and C. H. Williams), pp. 383-386. Elsevier, New York.

3. Macheroux, P., K. U. Schmidt, P. Steinerstauch, S. Ghisla, C. Colepicolo, R. Buntic and J. W. Hastings (1987) Purification of the yellow fluorescent protein from Vibrio fischeri and identity of the flavin chromophore. Biochem. Biophys. Res. Commun. 146, 101-106.

4. Daubner, S. C., A. M. Astorga, G. B. Leisman and T. O. Baldwin (1987) Yellow light emission of Vibrio fischeri strain Y-1: purification and characterization of the energy-accepting yellow fluorescent protein. Proc. Natl. Acad. Sci. USA 84, 8912-8916.

5. Karatani, H. and J. W. Hastings (1993) Two active forms of the accessory yellow fluorescence protein of the luminous bacterium V. fischeri. J. Photochem. Photobiol. B 18, 227-232.

6. Petushkov, V. N., B. G. Gibson and J. Lee (1995) The yellow bioluminescence bacterium, Vibrio fischeri $\mathrm{Y} 1$, contains a bioluminescence active riboflavin protein in addition to the yellow fluorescence FMN protein. Biochem. Biophys. Res. Commun. 211, 774-779.

7. Lee, J., I. B. C. Matheson, F. Müller, D. J. O'Kane, J. Vervoort and A. J. W. G. Visser (1991) The mechanism of bacterial bioluminescence. In Chemistry and Biochemistry of Flavoenzymes, Vol. II (Edited by F. Müller), pp. 109-151. CRC Press, Boca Raton, FL. 
8. Visser, A. J. W. G. and J. Lee (1980) Lumazine protein from the bioluminescent bacterium Photobacterium phosphoreum. A fluorescence study of the protein-ligand equilibrium. Biochemistry 19, 4366-4372.

9. Lee, J. (1993) Lumazine protein and the excitation mechanism in bacterial bioluminescence. Biophys. Chem. 48, 149-158.

10. Sirokman, G., T. Wilson and J. W. Hastings (1995) A bacterial luciferase reaction with a negative temperature coefficient attributable to protein-protein interaction. Biochemistry 34, 13074-13081.

11. Petushkov, V., B. G. Gibson and J. Lee (1995) Properties of recombinant fluorescent proteins from Photobacterium leiognathi and their interaction with luciferase intermediates. Biochemistry 34, 3300-3309.

12. van Hoek, A. and A. J. W. G. Visser (1992) $\mathrm{Cw}$ dye laser synchronously pumped by the third harmonic of a mode-locked cw Nd: YLF laser for tuneable blue and green excitation and picosecond time-correlated photon counting detection. Prö. SPIE Int. Soc. Opt. Eng. 1640, 325-329.

13. Leenders, R., M. Kooijman, A. van Hoek, C. Veeger and A. J. W. G. Visser (1993) Flavin dynamics in reduced flavodoxins: a time-resolved polarized fluorescence study. Eur. J. Biochem. 211, 37-45.

14. Beechem, J. M., E. Gratton, M. Ameloot, J. R. Knutson and L. Brand (1991) The global analysis of fluorescence intensity and anisotropy decay data: second-generation theory and programs. In Topics in Fluorescence Spectroscopy, Vol. 2 (Edited by J. R. Lakowicz), pp. 241-305. Plenum Press, New York.

15. Spencer, R. D. and G. Weber (1972) Thermodynamics and kinetics of the intramolecular complex in flavin adenine dinucle- otide. In Structure and Function of Oxidation Reduction Enzymes (Edited by $\AA$. Åkeson and A. Ehrenberg), pp. 393-399. Pergamon Press, Oxford.

16. Visser, A. J. W. G. (1984) Kinetics of stacking interactions in flavin adenine dinucleotide from time-resolved flavin fluorescence. Photochem. Photobiol. 40, 703-706.

17. Wahl, P. (1979) Analysis of fluorescence anisotropy decays by a least square method. Biophys. Chem. 10, 91-104.

18. Leenders, R., P. I. H. Bastiaens, R. Lunsche, A. van Hoek and A. J. W. G. Visser (1990) Rotational resolution of methyl-group substitution and anisotropic rotation of flavins as revealed by picosecond-resolved fluorescence depolarization. Chem. Phys. Lett. 165, 315-322.

19. Bastiaens, P. I. H., S. G. Mayhew, A. M. O’Nualláin, A. van Hoek and A. J. W. G. Visser (1991) Energy transfer between the two flavin chromophores of electron-transferring flavoprotein from Megasphaera elsdenii as inferred from time-resolved red-edge and blue-edge fluorescence spectroscopy. J. Fluoresc. 1, 95-103.

20. Bastiaens, P. I. H., A. van Hoek, W. F. Wolkers, J. C. Brochon and A. J. W. G. Visser (1992) Comparison of the dynamical structures of lipoamide dehydrogenase and glutathione reductase by time-resolved polarized flavin fluorescence. Biochemistry 31 , 7050-7060.

21. Visser, A. J. W. G., A. van Hoek, D. J. O'Kane and J. Lee (1989) Time-resolved fluorescence spectroscopy of lumazine protein from Photobacterium phosphoreum using synchrotron radiation. Eur. Biophys. J. 17, 75-85.

22. Weber, G. (1992). Protein Interactions, Chapter XVI. Chapman and Hall, New York, London. 